\title{
miR-589-5p is downregulated in prostate cancer and regulates tumor cell viability and metastasis by targeting CCL-5
}

\author{
LU JI, XI JIANG, FEI MAO, ZHIWANG TANG and BING ZHONG \\ Department of Urology, The Affiliated Huai'an No. 1 People's Hospital of \\ Nanjing Medical University, Huai'an, Jiangsu 223300, P.R. China
}

Received May 15, 2018; Accepted March 13, 2019

DOI: $10.3892 / \mathrm{mmr} .2019 .10334$

\begin{abstract}
Prostate cancer is one of the most common human malignancies, which represents a serious threat to health, and microRNAs (miRNAs/miRs) have been reported to be closely associated with the progression and development of prostate cancer. The present study aimed to investigate the expression patterns, functions and underlying mechanisms of miR-589-5p in prostate cancer. The results demonstrated that the expression levels of miR-589-5p were downregulated in prostate cancer tissues and cell lines. Overexpression of miR-589-5p inhibited cell viability, migration and invasion in prostate cancer cells. Subsequently, chemokine (C-C motif) ligand 5 (CCL-5) was identified as a direct target gene of miR-589-5p, which was highly expressed at the mRNA and protein levels in prostate cancer tissues and cells. Furthermore, CCL-5 mRNA was negatively correlated with miR-589-5p expression in prostate cancer tissues. Silencing CCL-5 promoted the apoptosis, and inhibited the migration and invasion of prostate cancer cells. Taken together, these results indicated that miR-589-5p may act as a tumor suppressor in prostate cancer by targeting CCL-5, thus suggesting that miR-589-5p may be a novel and reliable molecular marker for the diagnosis and prognosis of prostate cancer.
\end{abstract}

\section{Introduction}

Prostate cancer is one of the most common human malignancies and the second leading cause of cancer-associated mortality in men in western countries $(1,2)$. According to an updated estimate, prostate cancer has the highest incidence amongst male tumors in 2018, accounting for $19 \%$ of male malignant tumors, while this disease has been reported as the second leading cause of cancer-associated mortality (3). In recent years, the incidence of prostate cancer has greatly

Correspondence to: Dr Bing Zhong, Department of Urology, The Affiliated Huai'an No. 1 People's Hospital of Nanjing Medical University, 6 West Beijing Road, Huai'an, Jiangsu 223300, P.R. China E-mail: 15152569186@163.com

Key words: prostate cancer, miR-589-5p, CCL-5, viability, metastasis increased in China $(4,5)$. Furthermore, since the average life span is increasing and due to increased westernization of lifestyles, the incidence of prostate cancer is also increasing annually in China; notably, prostate cancer has been listed as one of the fastest growing tumors in China in the 21st century (6). Prostate cancer predominantly occurs in older men, and the median age of patients is $\sim 72$ years old; in addition, prostate cancer is the third most common major malignant tumor of the urogenital system, following bladder and renal tumors worldwide (7). The morphological characteristics and structure of prostate cancer are usually complex, and there is no obvious clinical symptom in the early stages; therefore, it is very difficult to make a clear diagnosis of prostate cancer (8). At present, the determination of prostate-specific antigen (PSA) in clinical serum samples is the most important diagnostic tool for prostate cancer. However, it has previously been reported that PSA is not the gold standard for diagnosis of prostate cancer. Although the sensitivity is high $(\sim 78.7 \%)$, the specificity is only $59.2 \%$, the false negative rate is $38-48 \%$ and the false positive rate is $25 \%$; therefore, prostate cancer can easily escape diagnosis or be misdiagnosed (9). Notably, rectal examination, imaging examination and other examination methods are also used in the clinical diagnosis of prostate cancer; however, these examinations do not achieve the desired results for the diagnosis of early prostate cancer, and may cause unnecessary injury and pain to patients $(10,11)$. At present, there are various methods for the treatment of prostate cancer, including surgical treatment, hormone therapy, radiotherapy, etc. Although these methods are widely used in clinical treatment, there are several limitations in the treatment and prognosis of prostate cancer (12-14). Therefore, research has recently paid more attention to the prevention and treatment of prostate cancer. Exploration of the molecular mechanisms underlying the development of prostate cancer, and identification of novel methods for the treatment of prostate cancer, have recently been focuses of research.

Numerous studies have reported that microRNAs (miRNAs/miRs) serve an irreplaceable role in the progression and development of prostate cancer, and have significant impacts on the biological behaviors of prostate cancer, including proliferation, differentiation, invasion and hormone dependence $(15,16)$. It has important application value and potential to provide reliable molecular markers for the diagnosis and prognosis of prostate cancer, which 
has unlimited potential as a target for treatment. miRNAs, which contain 17-25 nucleotides, suppress gene expression by binding to the 3'-untranslated regions (3'UTRs) of target genes, and have roles in various biological processes, including development, differentiation, cell proliferation and apoptosis (17-19). Distinct miRNA expression profiles have been identified in human prostate cancer tissues and cell lines, and it has been reported that miRNAs may act as either tumor suppressors or oncogenes in prostate cancer, depending on the functions of their target genes $(20,21)$. Therefore, it is important to investigate the abnormal expression of miRNAs and their functions in prostate cancer, in order to provide effective and novel therapeutic targets for anti-prostate cancer therapy.

miR-589-5p has previously been reported to be frequently abnormally expressed in numerous types of human cancer $(22,23)$. However, to the best of our knowledge, the expression and biological effects of miR-589-5p in prostate cancer have yet to be elucidated. Therefore, the present study aimed to investigate the expression levels of miR-589-5p in prostate cancer tissues and cell lines. In addition, the functions and underlying molecular mechanisms in prostate cancer were explored.

\section{Materials and methods}

Prostate cancer tissues. The present study was approved by the Ethical Committee of Nanjing Medical University (Huai'an, China). All patients provided written informed consent. A total of 30 prostate cancer tissues and corresponding adjacent normal tissues were obtained from patients who underwent surgical resection between June 2016 and April 2017 at the Affiliated Huai'an No. 1 People's Hospital of Nanjing Medical University (Huai'an, China); their average age was $45 \pm 7$ years. old; none of the patients received pre-operative chemotherapy or radiotherapy. All fresh tissues were stored at $-80^{\circ} \mathrm{C}$ for further study.

Cell culture and transfection. The DU-145 and PC3 human prostate cancer cell lines, and RWPE-1 normal prostate epithelial cell line were purchased from the Shanghai Institute of Biochemistry and Cell Biology of the Chinese Academy of Sciences (Shanghai, China). All cells were maintained in Dulbecco's modified Eagle's medium (Gibco; Thermo Fisher Scientific, Inc., Waltham, MA, USA) supplemented with $10 \%$ fetal bovine serum (FBS; Gibco; Thermo Fisher Scientific, Inc.), $100 \mathrm{U} / \mathrm{ml}$ penicillin, and $100 \mu \mathrm{g} / \mathrm{ml}$ streptomycin (Sigma-Aldrich; Merck KGaA, Darmstadt, Germany) at $37^{\circ} \mathrm{C}$ in an incubator containing $5 \% \mathrm{CO}_{2}$.

DU-145 and PC3 cells were seeded into 6-well plates at a density of $4 \times 10^{5}$ cells/well. Once cells reached $60-70 \%$ confluence, transfection with synthesized miR-589-5p mimics negative control (NC), miR-589-5p mimics, miR-589-5p inhibitors NC, miR-589-5p inhibitors, chemokine (C-C motif) ligand 5 (CCL-5) small interfering RNAs (si-CCL-5) and negative control siRNA (siRNA NC) were synthesized by Invitrogen; Thermo Fisher Scientific, Inc and was performed with Lipofectamine ${ }^{\circledR} 2000$ Reagent (Invitrogen; Thermo Fisher Scientific, Inc.) at a final concentration of $50 \mathrm{nM}$ for $24 \mathrm{~h}$, according to the manufacturer's protocols. The sequences were as follows: miR-589-5p mimics forward, 5'-TTTTGGAGT TTTTTTTGGTTTTT-3' and reverse, 5'-CAAAAACAAAAC CAA-AATCA-3'; miR-589-5p inhibitors forward, 5'-TGTTGG AGAGCCAAGTGGTATTT-3' and reverse, 5'-CCTGAACAG AACCGGACTCA-3'; miR-589-5p mimics NC or miR-589-5p inhibitors NC forward, 5'-GGCTGCATTGGCTGGCGA AACCCGUC-3' and reverse, 5'-ATGCGUGCCCTGCTGTTG C-TCCATGTCG-3'; si-CCL5 forward 5'-GCGTCGAGTTTG TCACGAGA-3', reverse 5'-TGACACTCCTTTACTGTGCT-3'. Transfected cells were cultured for 24,48 or $72 \mathrm{~h}$ under the same conditions as aforementioned.

$R N A$ isolation and reverse transcription-quantitative polymerase chain reaction ( $R T-q P C R)$. Total RNA was extracted from prostate cancer tissues and cell lines using TRIzol ${ }^{\circledR}$ reagent (Invitrogen; Thermo Fisher Scientific, Inc.) at $37^{\circ} \mathrm{C}$ for $30 \mathrm{~min}$, according to the manufacturer's protocol. For detection of miR-589-5p expression, TaqMan MicroRNA Reverse Transcription kit (Takara Biotechnology Co., Ltd., Dalian, China) was used to synthesize cDNA according to the manufacturer's protocol. The expression levels of miR-589-5p were examined using TaqMan MicroRNA PCR kit (Applied Biosystems; Thermo Fisher Scientific, Inc.), according to the manufacturer's protocol. To quantify CCL5 mRNA expression, the PrimeScript RT reagent kit (Applied Biosystems; Thermo Fisher Scientific, Inc.) was used to synthesize cDNA according to the manufacturer's protocol. Subsequently, CCL-5 mRNA was amplified using SYBR Premix Ex Taq (Applied Biosystems; Thermo Fisher Scientific, Inc.), according to the manufacturer's protocol. U6 and GAPDH were used as internal controls for miR-589-5p and CCL-5, respectively. The PCR primers used in the present study were as follows: miR-589-5p, forward 5'-CGAGGTCAG CGTGATTTCATGG-3', reverse 5'-TGTGTCCAAGTCCCA GCCAGAG-3'; U6, forward 5'-CTCGCTTCGGCAGCACA-3', reverse 5'-AACGCTTCACGAATTTGCGT-3'; CCL-5, forward 5'-CAGTCGTCTTTGTCACCCGA-3', reverse 5'-TGTAACTGC TGCTGTGTGGT-3'; and GAPDH, forward 5'-ACAACTTTG GTATCGTGGAAGG-3' and reverse 5'-GCCATCACGCCA CAGTTTC-3'. The thermocycling conditions comprised one cycle at $95^{\circ} \mathrm{C}$ for $30 \mathrm{sec}$, followed by 42 cycles of amplification $\left(95^{\circ} \mathrm{C}\right.$ for $3 \mathrm{sec}$ and $60^{\circ} \mathrm{C}$ for $\left.30 \mathrm{sec}\right)$. Fold-changes in miR-589-5p and CCL-5 mRNA expression were calculated using the $2^{-\Delta \Delta \mathrm{Cq}}$ method (24), and RT-qPCR reactions were performed in triplicate.

Cell viability assay. Cell viability was evaluated using the Cell Counting kit-8 (CCK-8) assay (Dojindo Molecular Technologies, Inc., Rockville, MD, USA). Briefly, DU-145 and PC 3 cells at a density of $3 \times 10^{3}$ cells/well were cultured in a 96-well plate and transfected with miR-589-5p mimics NC, miR-589-5p mimics, miR-589-5p inhibitors NC or miR-589-5p inhibitors. After 24,48 or $72 \mathrm{~h}$ at $37^{\circ} \mathrm{C}$ and $5 \% \mathrm{CO}_{2}, 10 \mu \mathrm{l}$ CCK- 8 reagent was added to each well and the cells were incubated for another $4 \mathrm{~h}$. The optical density was observed at a wavelength of $450 \mathrm{~nm}$ using a microplate reader (LNB, Shanghai, China).

Cell apoptosis. DU-145 and PC3 cells at a density of $1 \times 10^{5}$ cells/well were cultured in a 6 -well plate for $48 \mathrm{~h}$ post-transfection. Subsequently, the cells were detached, 
washed with PBS and centrifuged at 12,000 x $\mathrm{g}$ for $5 \mathrm{~min}$. The cells were then incubated with $500 \mu \mathrm{l}$ buffer, $5 \mu$ l fluorescein isothiocyanate-Annexin $\mathrm{V}$ and $5 \mu \mathrm{l}$ propidium iodide (PI) for $1 \mathrm{~h}$ at $37^{\circ} \mathrm{C}$, according to the manufacturer's protocols (Shanghai Yeasen Biotechnology, Co., Ltd., Shanghai, China). Cell apoptosis were analyzed using a flow cytometer (FACSCalibur; BD Biosciences, San Jose, CA, USA).

Wound-healing assay. For the wound-healing assay, DU-145 and PC3 cells were seeded into a 6-well plate at a density of $1 \times 10^{5}$ cells/well post-transfection for $24 \mathrm{~h}$. Once cell confluence reached $90 \%$, the cell monolayers were wounded in the surface of the plates using a $200 \mu \mathrm{l}$ pipette tip and washed with serum-free medium to remove floating cells. The cells were then cultured in a serum-free medium for cell recovery. Images of the cells were captured at 0 and $48 \mathrm{~h}$ under an inverted microscope.

Transwell invasion assay. The Transwell invasion assay was performed to evaluate the invasive capacity of prostate cancer cells. Following transfection for 24 h, DU-145 and PC3 cells at a density of $5 \times 10^{4}$ cells/well in $100 \mu \mathrm{l}$ serum-free medium were seeded into the upper chambers of a Transwell system (8 $\mu \mathrm{m}$; Corning Corporation, Corning, NY, USA), which were coated with Matrigel (BD Pharmingen; BD Biosciences). Culture medium containing 20\% FBS was added to the lower chambers. After $48 \mathrm{~h}$ at $37^{\circ} \mathrm{C}$, the cells remaining on the upper compartments were removed, whereas the invaded cells were fixed with $4 \%$ paraformaldehyde at room temperature for 30 min and stained with $10 \%$ crystal violet at room temperature for $15 \mathrm{~min}$. Cell numbers were obtained from five fields per membrane under a light microscope (Olympus Corporation, Tokyo, Japan). The following equations were used to analyze findings: Invasion rate $(\%)=$ invasive cells/total cells.

Target prediction for miR-589-5p and luciferase reporter assay. The candidate target genes of miR-589-5p were analyzed using the miRNA target prediction programs PicTar (pictar. mdc-berlin.de/) and TargetScan (www.targetscan.org/). The 3'UTR sequences of CCL-5 containing the putative binding site for miR-589-5p were amplified from human genomic DNA, and cloned into the pGL3 vector (Promega Corporation, Madison, WI, USA), in order to obtain the recombinant vectors pGL3-CCL-5-wild-type (wt) and pGL3-CCL-5-mutant (mut). Subsequently, DU-145 and PC3 cells $\left(1 \times 10^{5}\right)$ were plated in 48-well plates for $24 \mathrm{~h}$, and psiCheck-2 with the wild-type (WT) or mutant (MUT) 3'-untranslated region (UTR) of CCL-5 was cotransfected with miR-589-5p mimics or miR-NC at a concentration of $50 \mathrm{nM}$ using Lipofectamine ${ }^{\circledR} 2000$ (Invitrogen; Thermo Fisher Scientific, Inc.). After transfection for $48 \mathrm{~h}$, cells were collected and subjected to a luciferase assay using the Dual-Luciferase Reporter Assay kit according to the manufacturer's protocol (Promega Corporation).

Western blotting. Total protein was extracted from tissues and transfected cells using radioimmunoprecipitation assay buffer (Beijing Solarbio Science \& Technology Co., Ltd., Beijing, China). Subsequently, the supernatants were collected following centrifugation at $12,000 \times \mathrm{g}$ for $20 \mathrm{~min}$ at $4^{\circ} \mathrm{C}$, and the protein concentrations were determined using a Bicinchoninic
Acid Protein Assay kit (Vazyme, Piscataway, NJ, USA). Loading buffer was added to the supernatant of samples and the proteins were denatured at $100^{\circ} \mathrm{C}$ for $5 \mathrm{~min}$. Equal amounts of protein $(30 \mu \mathrm{g})$ were then separated by $12 \%$ SDS-PAGE and transferred onto polyvinylidene difluoride membranes. The membranes were blocked with 5\% non-fat milk for at room temperature for $1 \mathrm{~h}$, washed four times with Tris-buffered saline plus $0.1 \%$ Tween (TBST; $15 \mathrm{~min} /$ wash), and incubated overnight at $4^{\circ} \mathrm{C}$ with primary antibodies. The following primary antibodies were used: Anti-CCL-5 (cat. no. ab189841; 1:1,000), B-cell lymphoma 2 (Bcl-2)-associated X protein (Bax; cat. no. ab32503; 1:1,000), Bcl-2 (cat. no. ab196495; 1:1,000), caspase-3 (cat. no. ab90437; 1:1,000), caspase-9 (cat. no. ab25758; 1:1,000), matrix metalloproteinase (MMP)-2 (cat. no. ab37150; 1:1,000), MMP-9 (cat. no. ab73734; 1:1,000) and GAPDH (cat. no. ab9485; 1:1,000) (all from Abcam, Cambridge, UK). Subsequently, the membranes were washed with TBST three times (15 min/wash) and incubated with horseradish peroxidase-conjugated secondary antibodies (cat. no. ab6721; 1:2,000; Abcam) for $2 \mathrm{~h}$ at room temperature. Protein bands were examined using the Bio-Rad Imaging system (Hercules, CA, United States) with an enhanced chemiluminescence western blotting substrate kit (ProteinTech Group, Inc., Wuhan, China) and the results were measured using ImageJ software 1.48 (National Institutes of Health, Bethesda, MD, USA). GAPDH were used as a loading control.

Statistical analysis. All data are expressed as the means \pm standard deviation, and all experiments were repeated at least three times. Statistical analyses were performed using SPSS 20.0 (IBM Corp., Armonk, NY, USA). Multiple group comparisons were conducted using one-way analysis of variance and Tukey's test. Student's t-test was used to compare only two groups. The association between CCL-5 and miR-589-5p expression in prostate cancer was evaluated using Spearman's correlation coefficient. $\mathrm{P}<0.05$ was considered to indicate a statistically significant difference.

\section{Results}

miR-589-5p is downregulated in prostate cancer tissues and cell lines. In order to explore the role of miR-589-5p in prostate cancer, RT-qPCR was performed to evaluate its expression in prostate cancer tissues and cells. As presented in Fig. 1A, miR-589-5p was markedly downregulated in prostate cancer tissues compared with in corresponding adjacent normal tissues. In addition, the expression levels of miR-589-5p were examined in prostate cancer cells (DU-145 and PC3) and in RWPE normal prostate epithelial cells. The results demonstrated that, compared with in RWPE cells, the expression levels of miR-589-5p were significantly decreased in DU-145 and PC3 cells (Fig. 1B). These findings indicated that miR-589-5p may act as a tumor suppressor in prostate cancer progression and development.

miR-589-5p inhibits viability and induces apoptosis of prostate cancer cells. The present study demonstrated that miR-589-5p was downregulated in prostate cancer cells. To further explore the role of miR-589-5p in the progression and development of prostate cancer, miR-589-5p mimics NC, miR-589-5p mimics, 

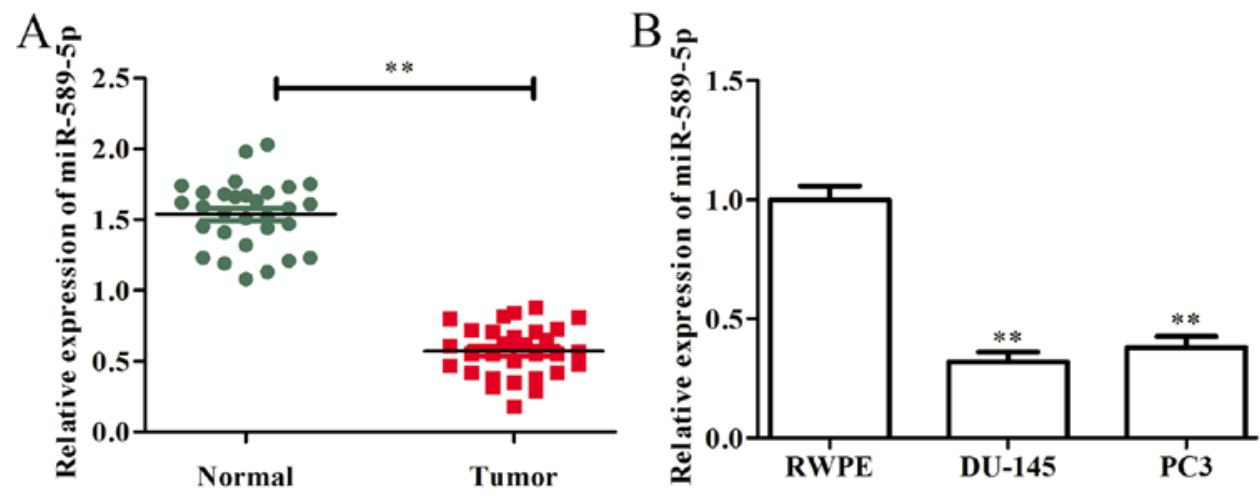

Figure 1. miR-589-5p is downregulated in prostate cancer tissues and cells. (A) miR-589-5p expression was detected in 30 prostate cancer tissues and matched adjacent normal tissues using RT-qPCR. (B) miR-589-5p expression was detected in prostate cancer and normal prostate epithelial cells using RT-qPCR. Data are presented as the means \pm standard deviation of three independent experiments, each experiment was performed in triplicate. ${ }^{* *} \mathrm{P}<0.01$ compared with the control group. miR-589-5p, microRNA-589-5p; RT-qPCR, reverse transcription-quantitative polymerase chain reaction.

miR-589-5p inhibitors NC and miR-589-5p inhibitors were transfected into DU-145 and PC3 cells. After transfection for $48 \mathrm{~h}$, transfection efficiency was determined by RT-qPCR. As shown in Fig. 2A, the expression levels of miR-589-5p were significantly upregulated following transfection with miR-589-5p mimics, whereas they were downregulated post-transfection with miR-589-5p inhibitors, as compared with in the NC-transfected DU-145 and PC 3 cells.

CCK-8 assay was performed to evaluate the effects of miR-589-5p on the viability of DU-145 and PC 3 cells; the results revealed that the viability of DU-145 and PC3 cells transfected with miR-589-5p mimics was markedly suppressed compared with in cells transfected with the NC, whereas miR-589-5p inhibitors significantly promoted the viability of DU-145 and PC3 cells compared with the NC group (Fig. 2B).

The induction of apoptosis is a critical process through which miRNAs exert anticancer effects (25). Therefore, the association between miR-589-5p and DU-145 and PC 3 cell apoptosis was revealed by flow cytometry. Post-transfection with miR-589-5p mimics NC, miR-589-5p mimics, miR-589-5p inhibitors NC and miR-589-5p inhibitors, PI and Annexin V double staining was conducted to evaluate apoptosis. As shown in Fig. 2C, miR-589-5p mimics promoted apoptosis, whereas miR-589-5p inhibitors inhibited apoptosis of DU-145 and PC3 cells compared with in the NC groups.

Tumor cell apoptosis is mediated by various molecules that inhibit (e.g. Bcl-2) or induce (e.g. Bax and caspases) cell death (26). Therefore, the present study detected the protein expression levels of Bcl-2, Bax, caspase-3 and caspase-9. The results demonstrated that pro-apoptotic proteins, including Bax, caspase- 3 and caspase-9, were increased, whereas the expression levels of the anti-apoptotic protein Bcl-2 were significantly decreased in DU-145 and PC3 cells transfected with miR-589-5p mimics (Fig. 2D). Conversely, transfection with miR-589-5p inhibitors exerted the opposite effects on DU-145 and PC 3 cells.

miR-589-5p suppresses the migration and invasion of prostate cancer cells. Wound-healing and Transwell invasion assays were performed to investigate the effects of miR-589-5p on migration and invasion of DU-145 and PC 3 cells transfected with miR-589-5p mimics NC, miR-589-5p mimics, miR-589-5p inhibitors NC and miR-589-5p inhibitors. The results indicated that the migratory capacity of DU-145 and PC 3 cells transfected with miR-589-5p mimics was markedly suppressed compared with cells transfected with miR-589-5p mimics NC, whereas transfection of DU-145 and PC3 cells with miR-589-5p inhibitors notably increased cell migration into the wound (Fig. 3A). Furthermore, a Transwell invasion assay revealed that miR-589-5p mimics markedly suppressed DU-145 and PC 3 cell invasion. However, when DU-145 and PC3 cells were transfected with miR-589-5p inhibitors, invasion was significantly increased compared with in cells in the miR-589-5p inhibitors NC group (Fig. 3B).

During the progression and development of metastasis in prostate cancer, MMPs serve a significant role (27). In the present study, the protein expression levels of MMP-2 and MMP-9 were detected in DU-145 and PC3 cells transfected with miR-589-5p mimics NC, miR-589-5p mimics, miR-589-5p inhibitors $\mathrm{NC}$ and miR-589-5p inhibitors. The results indicated that miR-589-5p mimics markedly suppressed the protein expression levels of MMP-2 and MMP-9, whereas the expression levels of MMP-2 and MMP-9 were significantly enhanced following transfection with miR-589-5p inhibitors (Fig. 3C).

CCL-5 is a direct target of miR-589-5p in prostate cancer cells. This study aimed to explore the molecular mechanisms underlying the effects of miR-589-5p on prostate cancer cell viability, apoptosis, migration and invasion. Firstly, PicTar and TargetScan were used to predict the possible target gene of miR-589-5p; the results demonstrated that CCL-5 may be a potential target gene of miR-589-5p (Fig. 4A). Furthermore, a luciferase reporter assay was performed to confirm whether miR-589-5p binds to the 3'UTR of CCL-5; the results demonstrated that miR-589-5p directly binds to the 3 'UTR of CCL-5 following co-transfection with miR-589-5p mimics and luciferase reporter vector; ectopic expression of miR-589-5p decreased the luciferase activity of CCL-5 3'UTR-wt, whereas it did not affect that of CCL-5 3'UTR-mut (Fig. 4B).

To further confirm whether CCL-5 was a direct target gene of miR-589-5p, RT-qPCR and western blotting were performed to evaluate the mRNA and protein expression levels of CCL-5 

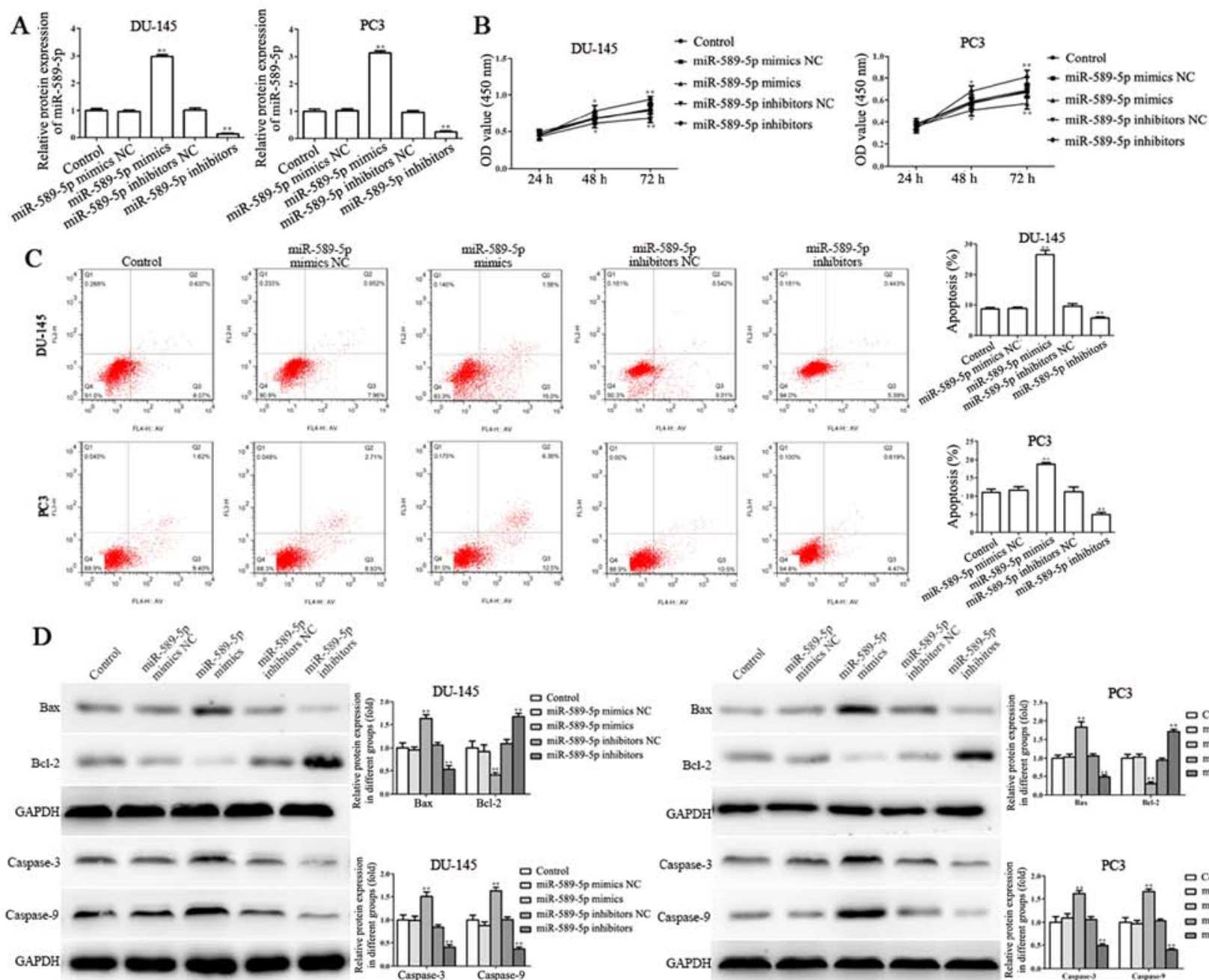

Contrel miR-589.5p mimies NC miR-589.5p intibitioes NC
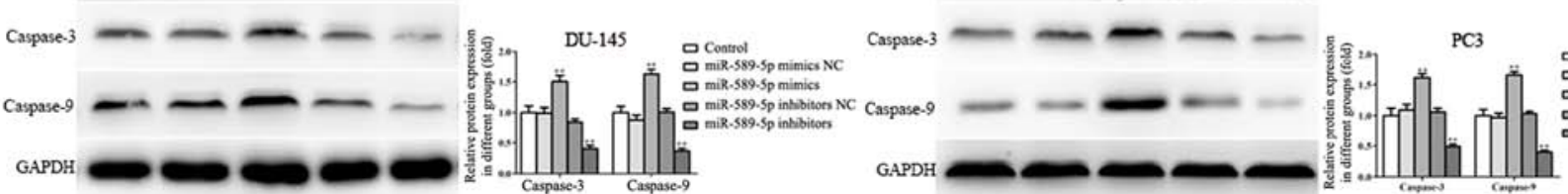

DControl mik-589.5p mimics $\mathrm{NC}$ mil-589-5p intibitions $N$

Figure 2. miR-589-5p inhibits viability and induces apoptosis of prostate cancer cells. (A) Transfection efficiency was assessed by reverse transcription-quantitative polymerase chain reaction post-transfection with miR-589-5p mimics or miR-589-5p inhibitors in DU-145 and PC3 cells. (B) Viability of DU-145 and PC3 cells was determined post-transfection with miR-589-5p mimics or miR-589-5p inhibitors for 24,48 and 72 h using the Cell Counting kit-8 assay. (C) Apoptosis assays were performed by flow cytometry post-transfection with miR-589-5p mimics or miR-589-5p inhibitors for 48 h in DU-145 and PC3 cells. (D) Protein expression levels of Bax, Bcl-2, caspase-3 and caspase-9 were examined in DU-145 and PC3 cells transfected with miR-589-5p mimics or miR-589-5p inhibitors for $48 \mathrm{~h}$; band intensity was semi-quantified using ImageJ software. Data are presented as the means \pm standard deviation of three independent experiments, each experiment was performed in triplicate. ${ }^{*} \mathrm{P}<0.05,{ }^{* *} \mathrm{P}<0.01$ compared with the control group. Bax, Bcl-2-associated $\mathrm{X}$ protein; Bcl-2, B-cell lymphoma 2; miR-589-5p, microRNA-589-5p; NC, negative control; OD, optical density.

in DU-145 and PC3 cells post-transfection with miR-589-5p mimics NC, miR-589-5p mimics, miR-589-5p inhibitors NC and miR-589-5p inhibitors. The results revealed that overexpression of miR-589-5p significantly decreased the mRNA and protein expression levels of CCL-5, whereas knockdown of miR-589-5p increased the mRNA and protein expression levels of CCL-5 in DU-145 and PC3 cells (Fig. 4C and D). These findings strongly indicated that CCL-5 was the direct target gene of miR-589-5p.

CCL-5 is upregulated in prostate cancer tissues and cells. To further evaluate the role of CCL-5 in prostate cancer, the mRNA and protein expression levels of CCL-5 were determined in prostate cancer tissues and cells by RT-qPCR and western blotting. As shown in Fig. 5A-D, the mRNA and protein expression levels of CCL-5 were significantly upregulated in prostate cancer tissues and cells compared with in the corresponding adjacent normal tissues and normal prostate epithelial cells, respectively. In addition, the association between CCL-5 and miR-589-5p expression in prostate cancer was evaluated using Spearman's correlation analysis; the results demonstrated that CCL-5 mRNA expression was negatively correlated with miR-589-5p expression in prostate cancer tissues (Fig. 5E).

Knockdown of CCL-5 suppresses viability, migration and invasion, and induces apoptosis of prostate cancer cells. In order to investigate the role of CCL-5 in the regulation of viability, apoptosis, migration and invasion in DU-145 and PC3 cells, si-CCL-5 was transfected into DU-145 and PC3 cells using Lipofectamine ${ }^{\circledR} 2000$ Reagent. Subsequently, RT-qPCR was performed to determine transfection efficiency; the results demonstrated that si-CCL-5 markedly inhibited the mRNA and protein expression levels of CCL-5 in DU-145 and PC3 cells (Fig. 6A and B). In addition, flow cytometry was performed to assess the effects of CCL-5 on 


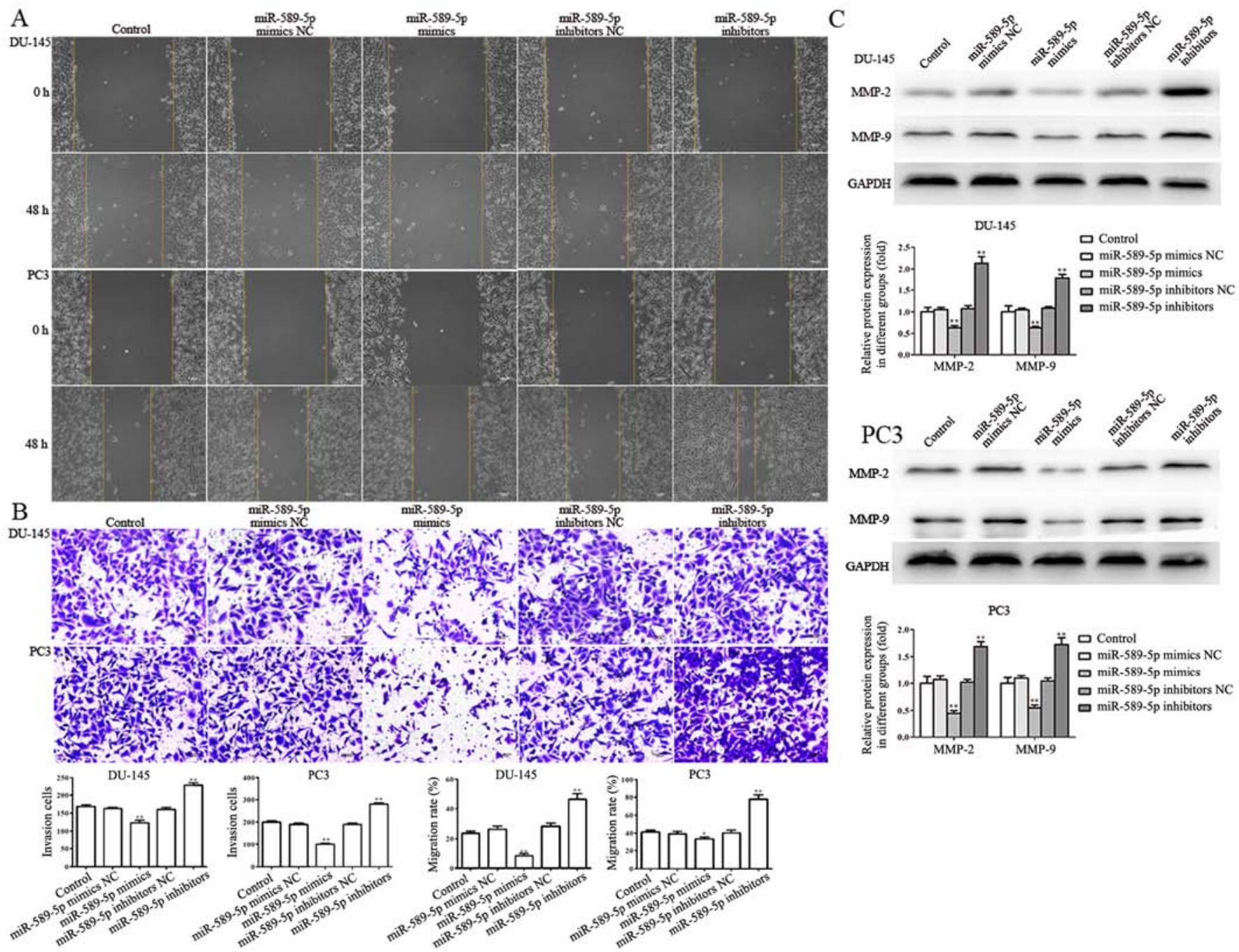

Figure 3. miR-589-5p suppresses the migration and invasion of prostate cancer cells. (A) Migratory abilities of DU-145 and PC 3 cells transfected with miR-589-5p mimics or miR-589-5p inhibitors for $48 \mathrm{~h}$ were examined by wound-healing assay (magnification, $\mathrm{x} 200$ ). (B) Invasive abilities of DU-145 and PC3 cells transfected with miR-589-5p mimics or miR-589-5p inhibitors for $48 \mathrm{~h}$ were evaluated by Transwell invasion assay (magnification, $\mathrm{x} 200$ ). (C) Protein expression levels of MMP-2 and MMP-9 were examined in DU-145 and PC3 cells transfected with miR-589-5p mimics or miR-589-5p inhibitors for 48 h; band intensity was semi-quantified using ImageJ software. Data are presented as the means \pm standard deviation of three independent experiments, each experiment was performed in triplicate. ${ }^{*} \mathrm{P}<0.05,{ }^{* *} \mathrm{P}<0.01$ compared with the control group. miR-589-5p, microRNA-589-5p; MMP, matrix metalloproteinase; NC, negative control.

the apoptosis of DU-145 and PC3 cells. The results verified that si-CCL-5 markedly promoted the apoptosis of DU-145 and PC3 cells; the proportions of apoptotic cells were $15.67 \pm 3.87$ and $19.27 \pm 4.29$ compared with in the control group (7.86 \pm 4.13 and $11.17 \pm 5.31$ ), respectively (Fig. 6C). Furthermore, wound-healing, and Transwell invasion assays were performed to examine the effects of CCL-5 on the migration and invasion of DU-145 and PC 3 cells. As shown in Fig. 6D and E, it was revealed that CCL-5, as a target gene for miR-589-5p, may serve an essential role in the progression and development of prostate cancer.

\section{Discussion}

Prostate cancer is the most common malignancy in men, and is the second leading cause of cancer-associated mortality in men. In addition, the incidence of prostate cancer increases with age (28). There are no symptoms during the early stage of prostate cancer, and symptoms occur only when the tumor obstructs the urethra or bladder neck (29). Therefore, early diagnosis and treatment are important factors in determining the cure rates and prognosis of tumor patients, and searching for reliable and effective molecular markers to guide the diagnosis and prognosis of prostate cancer is a popular topic in clinical research. miRNAs are non-coding short-chain RNAs, which have significant roles as oncogenes and tumor suppressor genes in the development of various types of cancer $(30,31)$. It has previously been reported that miR-589-5p acts as a tumor suppressor in non-small cell lung cancer (22). In addition, the long non-coding RNA (lncRNA) putative fatty acid-binding protein 5-like protein 3 (FABP5P3) can bind to miR-589-5p, which serves a tumor-enhancing role in hepatocellular carcinoma; the lncRNA FABP5P3/miR-589-5p/zinc finger MYND-type containing 19 axis contributes to hepatocellular carcinoma cell proliferation, migration and invasion (23). In addition, miR-589-5p can regulate mitogen-activated protein kinase kinase kinase 8 expression in hepatocellular carcinoma (32). However, the role and mechanism of miR-589-5p 
A

B

miR-589-5p 3' GAGUCUGUCUGCACCAAGAGU

CCL-5 5' CGGCUUACCAUUAAAGUUCUCAA
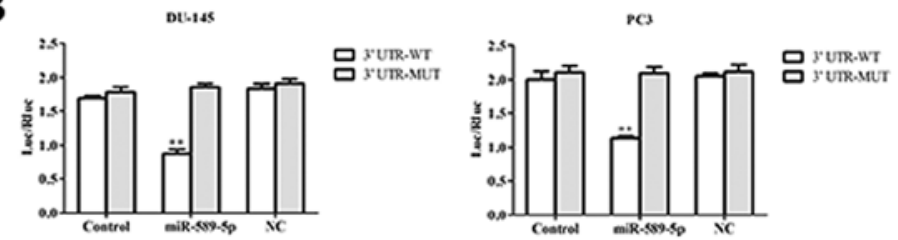

$\mathrm{C}$

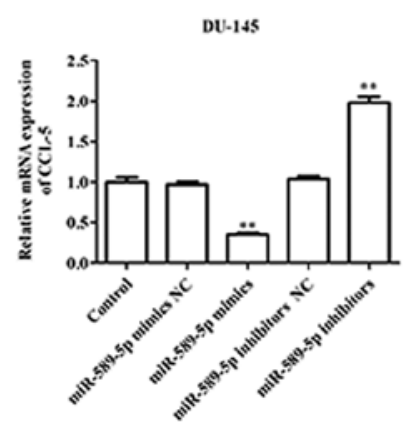

$\mathrm{D}$
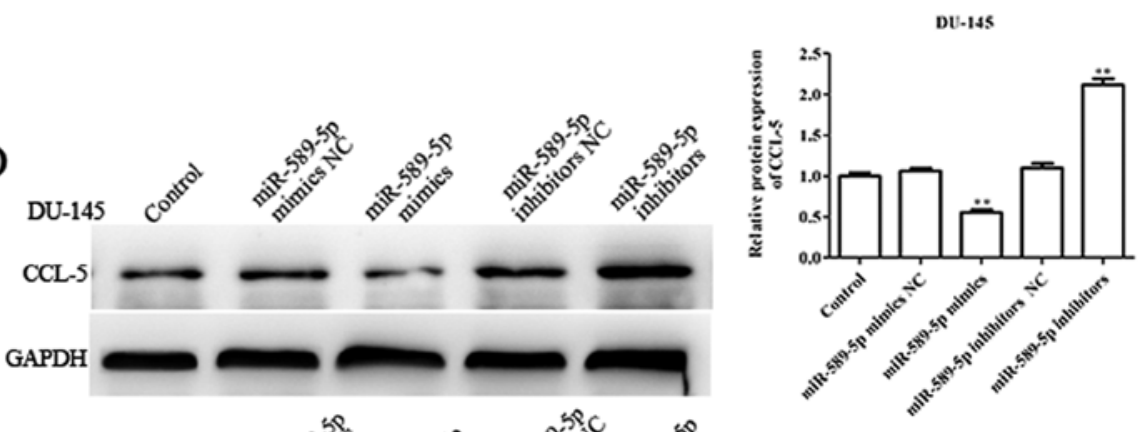

$\mathrm{PCS}$
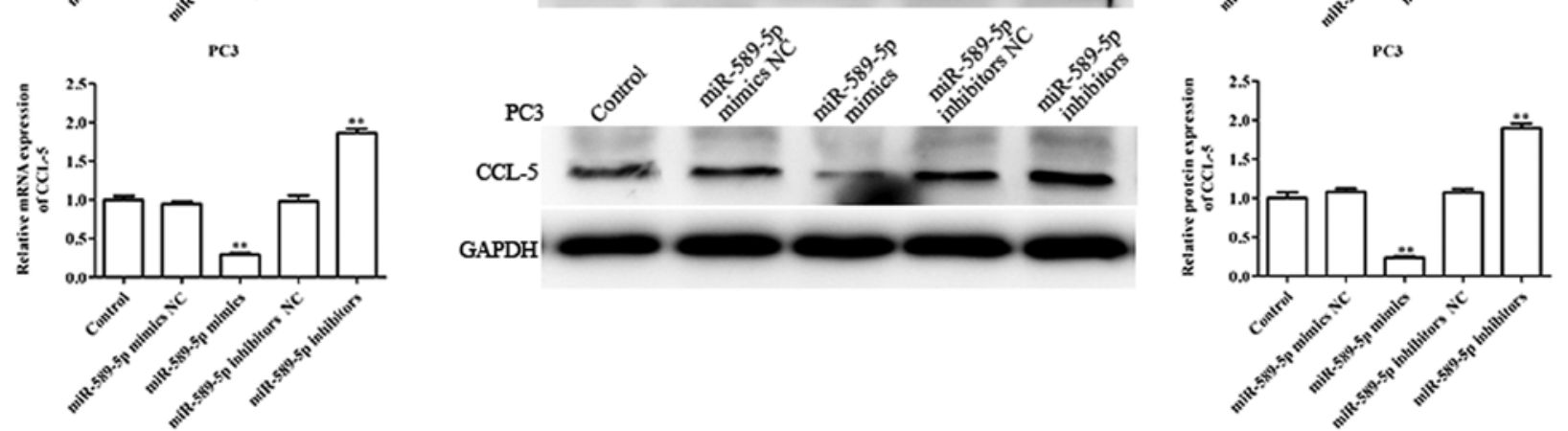

Figure 4. CCL-5 is a direct target gene of miR-589-5p in prostate cancer cells. (A) Prediction of the binding between miR-589-5p and CCL-5, as determined by TargetScan. (B) Luciferase reporter assays were performed to verify the binding of miR-589-5p to the 3'UTR of CCL-5. (C) Reverse transcription-quantitative polymerase chain reaction and (D) western blotting were performed to evaluate the mRNA and protein expression levels of CCL-5 following transfection with miR-589-5p mimics or miR-589-5p inhibitors. Band intensity was semi-quantified using ImageJ software. Data are presented as the means \pm standard deviation of three independent experiments, each experiment was performed in triplicate. ${ }^{* *} \mathrm{P}<0.01$ compared with the control group. $3^{\prime} \mathrm{UTR}, 3^{\prime}$-untranslated region; CCL-5, chemokine (C-C motif) ligand 5; miR-589-5p, microRNA-589-5p; MUT, mutant; NC, negative control; Rluc, Renilla luciferase; WT, wild-type.

A

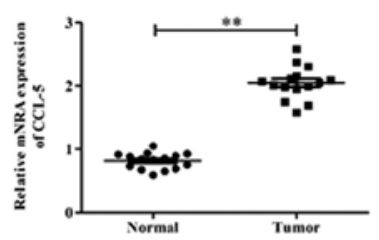

B

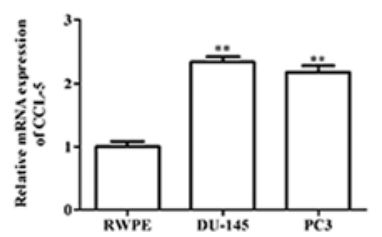

$\mathrm{C}$
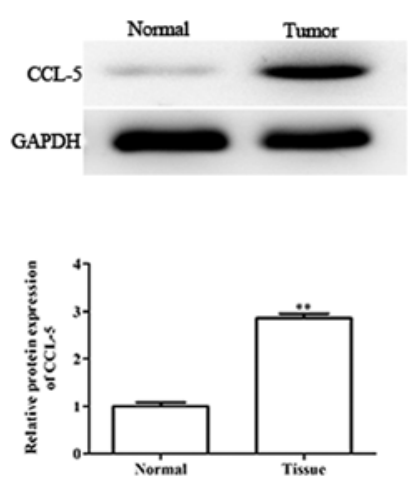

$\mathrm{D}$

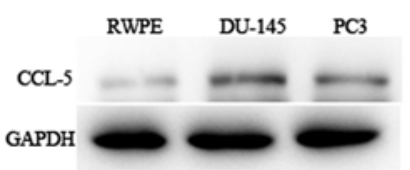

E

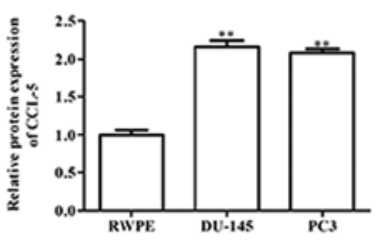

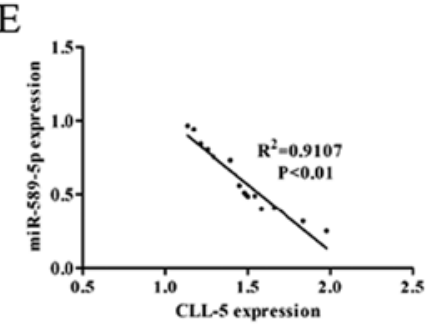

Figure 5. CCL-5 is upregulated in prostate cancer tissues and cells. (A) RT-qPCR and (B) western blotting were performed to detect the mRNA and protein expression levels of CCL-5 in 15 prostate cancer tissues and matched adjacent normal tissues. (C) RT-qPCR and (D) western blotting were performed to detect the mRNA and protein expression levels of CCL-5 in prostate cancer cell lines and normal prostate epithelial cells. Band intensity was semi-quantified using ImageJ software. (E) Analysis of the association between miR-589-5p and CCL-5 expression in prostate cancer tissues. Data are presented as the means \pm standard deviation of three independent experiments, each experiment was performed in triplicate. ${ }^{* *} \mathrm{P}<0.01$ compared with the control group. CCL-5, chemokine (C-C motif) ligand 5; miR-589-5p, microRNA-589-5p; NC, negative control; RT-qPCR, reverse transcription-quantitative polymerase chain reaction.

in prostate cancer remain elusive. The present study confirmed that the expression levels of miR-589-5p in prostate cancer tissues and cell lines were abnormally downregulated, thus suggesting that miR-589-5p may act as a tumor suppressor involved in the progression and development of prostate cancer. 
A
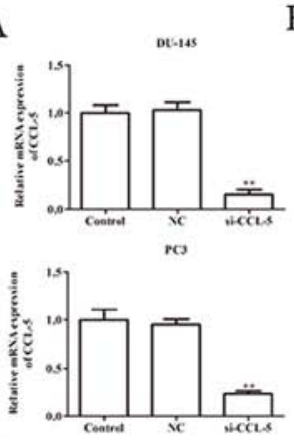

$\mathrm{C}$
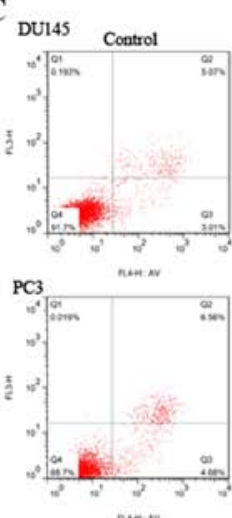

B
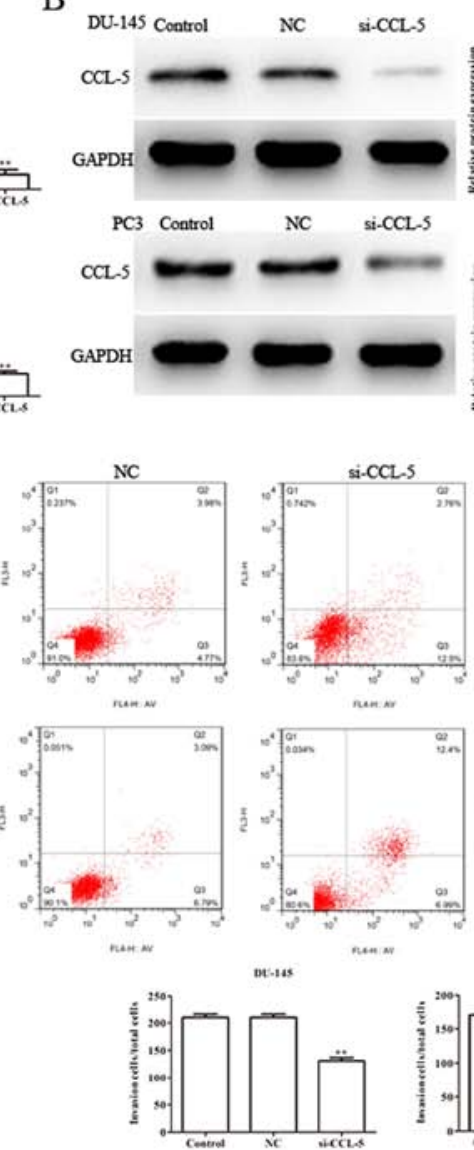
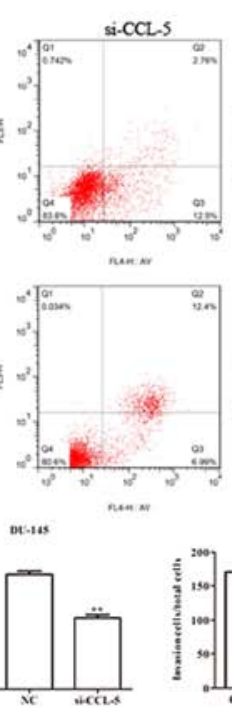

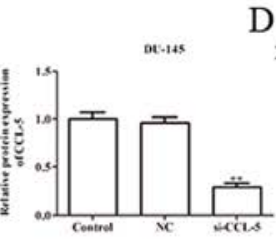

ros
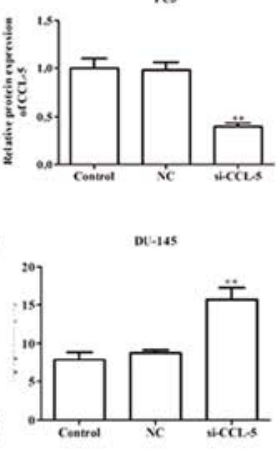

ro $\quad \mathrm{E}$

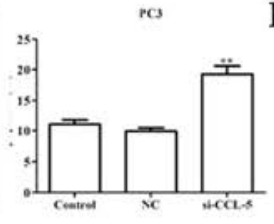

ro

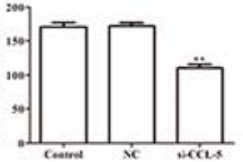

D

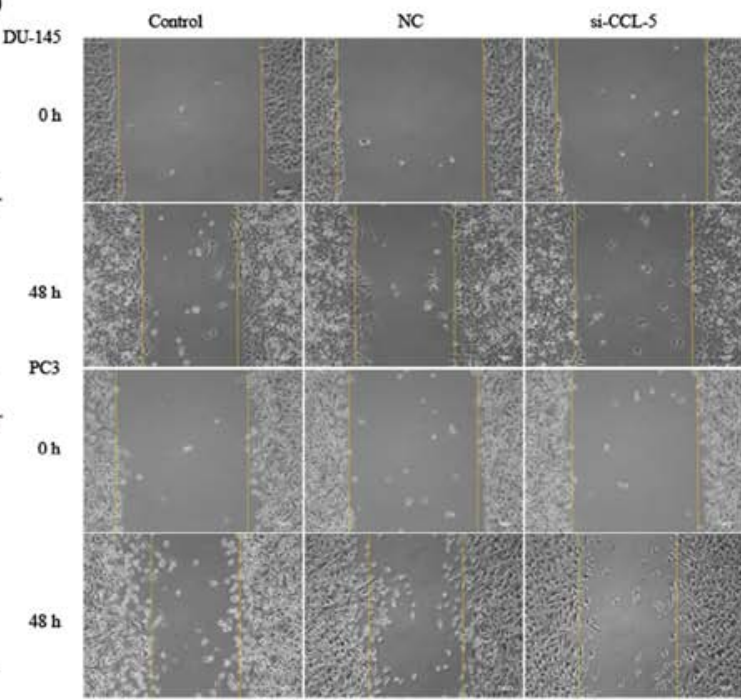

E

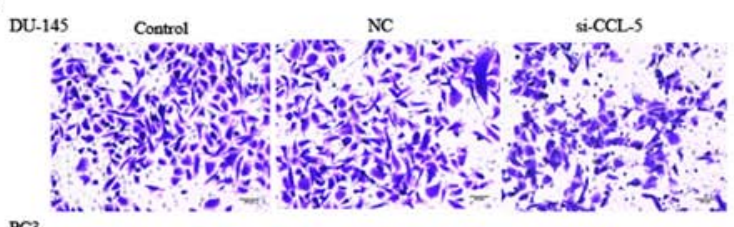

PC3

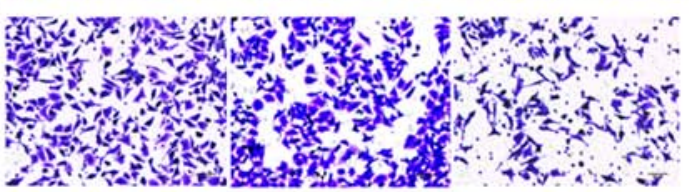

Figure 6. Silencing of CCL-5 suppresses viability, migration and invasion, and induces apoptosis of prostate cancer cells. (A) Reverse transcription-quantitative polymerase chain reaction and (B) western blotting were performed to assess knockdown efficiency post-transfection with si-CCL-5 in DU-145 and PC 3 cells. (C) Apoptosis assays were performed using flow cytometry post-transfection with si-CCL-5 for 48 h in DU-145 and PC3 cells. (D) Migratory abilities of DU-145 and PC3 cells transfected with si-CCL-5 for $48 \mathrm{~h}$ were examined by wound-healing assay (magnification, x200). (E) Invasive abilities of DU-145 and PC3 cells transfected with si-CCL-5 for $48 \mathrm{~h}$ were evaluated by Transwell invasion assay (magnification, $\mathrm{x} 200$ ). Data are presented as the means \pm standard deviation of three independent experiments, each experiment was performed in triplicate. ${ }^{* *} \mathrm{P}<0.01$ compared with the control group. CCL-5, chemokine (C-C motif) ligand 5; miR-589-5p, microRNA-589-5p; NC, negative control; si, small interfering RNA.

Apoptosis is a programmed cell death controlled by intracellular signals (33). There are two classic apoptotic pathways: The death receptor-mediated extrinsic pathway and the mitochondria-mediated intrinsic pathway. Alterations in mitochondrial outer membrane permeabilization can result in the release of various pro-apoptotic factors from the intermembrane space to the cytoplasm, which further activates caspases and induces cell apoptosis (34). Furthermore, upregulation of the Bax:Bcl-2 ratio results in the release of cytochrome $c$ from the mitochondria and activates mitochondria-dependent caspases to induce apoptotic cell death (35). In the present study, overexpression of miR-589-5p markedly inhibited the viability of DU-145 and PC3 prostate cancer cells, whereas knockdown of miR-589-5p promoted DU-145 and PC3 cell viability. In addition, miR-589-5p mimics increased the protein expression levels of Bax, caspase- 3 and caspase-9, and decreased the protein expression levels of Bcl-2 in DU-145 and PC3 cells, whereas miR-589-5p inhibitors exhibited the opposite effects.

Tumor invasion and metastasis are biological characteristics of malignant tumors, and are the main causes of mortality in patients with cancer (36). The present results confirmed the inhibitory role of miR-589-5p in the invasion and metastasis of
DU145 and PC 3 cells by wound-healing and Transwell invasion assays. It has been reported that tumor invasion and metastasis are a series of complex, multi-step and multi-factor dynamic process between tumor cells and host cells or the extracellular matrix (ECM) $(37,38)$. A series of tissue barriers are encountered in the process of tumor invasion and metastasis; these barriers are made up of basement membrane, mesenchyme and matrix in the ECM. Among the enzymes involved in the destruction of the ECM, MMPs are the most closely associated with tumor invasion and metastasis (39-41). The present study revealed that miR-589-5p mimics significantly suppressed the expression levels of MMP-2 and MMP-9, whereas the expression levels of MMP-2 and MMP-9 were promoted following transfection with miR-589-5p inhibitors.

According to the strict complementary nature of base pairs, miRNAs can target and bind mRNAs, and can inhibit the expression of target genes through degrading target mRNAs or inhibiting the translation progress. These functions of miRNAs participate in the progression and development of cancer (42). CCL-5 is a chemokine that belongs to the chemokine C-C family, which is mainly expressed in T lymphocytes, macrophages, platelets, synovial fibroblasts, renal tubular epithelial cells and certain types of tumor cells (43). Previous studies have 
detected CCL-5 expression in tissues and serum samples from patients with pancreatic cancer, lung cancer, melanoma, etc., and it is correlated with disease progression $(44,45)$. In addition, CCL-5, as a target gene, takes part in the development of numerous types of tumor $(46,47)$. In this study, TargetScan and PicTar were used to analyze the target genes of miR-589-5p, and a luciferase reporter assay confirmed that CCL-5 may be a potential candidate of miR-589-5p. Furthermore, the role of CCL-5 in the progression and development of prostate cancer was evaluated. Upregulation of CCL-5 was observed in prostate cancer tissues and cell lines, and si-CCL-5 had inhibitory effects on viability, apoptosis, migration and invasion in DU145 and PC3 cells. In addition, the mRNA expression levels of CCL-5 were negatively correlated with miR-589-5p expression in prostate cancer tissues. These results confirmed that CCL-5 acts as a target gene of miR-589-5p and may serve an important role in the progression and development of prostate cancer.

In conclusion, the present study confirmed that miR-589-5p was downregulated in prostate cancer tissues and cell lines. In addition, it was suggested that miR-589-5p may function as a potential tumor suppressor in prostate cancer to inhibit cell growth and metastasis by targeting CCL-5. Therefore, miR-589-5p may be considered a reliable molecular marker for the diagnosis and prognosis of prostate cancer. This study presented preliminary research on the antitumor effects of miR-589-5p in prostate cancer. At present, it is unclear whether miR-589-5p has anti-tumor effects in other types of cancer and the underlying mechanism requires further investigation; therefore, we aim to further explore the signaling pathways that are involved in CCL-5-mediated regulation of cell viability and metastasis in future studies.

\section{Acknowledgements}

Not applicable.

\section{Funding}

No funding was received.

\section{Availability of data and materials}

The datasets used and/or analyzed during the current study are available from the corresponding author on reasonable request.

\section{Authors' contributions}

BZ conceived and designed the present study. XJ and FM performed the experiments. LJ and ZT acquired and analyzed the data. All authors have read and approved the manuscript and agree to be accountable for all aspects of the research in ensuring that the accuracy and integrity of any part of the work are appropriately investigated and resolved.

\section{Ethics approval and consent to participate}

This study was approved by the Ethical Committee of Nanjing Medical University. All patients provided written informed consent.

\section{Patient consent for publication}

Not applicable.

\section{Competing interests}

The authors declare that they have no competing interests.

\section{References}

1. Kojima K, Fujita Y, Nozawa Y, Deguchi T and Ito M: miR-34a attenuates paclitaxel-resistance of hormone-refractory prostate cancer PC3 cells through direct and indirect mechanisms. Prostate 70: 1501-1512, 2010

2. Viticchiè G, Lena AM, Latina A, Formosa A, Gregersen LH, Lund AH, Bernardini S, Mauriello A, Miano R, Spagnoli LG, et al: miR-203 controls proliferation, migration and invasive potential of prostate cancer cell lines. Cell Cycle 10: 1121-1131, 2011.

3. Guan H, You Z, Wang C, Fang F, Peng R, Mao L, Xu B and Chen M: MicroRNA-200a suppresses prostate cancer progression through BRD4/AR signaling pathway. Cancer Med 8: 1474-1485, 2019.

4. Fan $\mathrm{H}$ and Zhang YS: miR-490-3p modulates the progression of prostate cancer through regulating histone deacetylase 2. Eur Rev Med Pharmacol Sci 23: 539-546, 2019.

5. Zhang FB, Du Y, Tian Y, Ji ZG and Yang PQ: miR-1299 functions as a tumor suppressor to inhibit the proliferation and metastasis of prostate cancer by targeting NEK2. Eur Rev Med Pharmacol Sci 23: 530-538, 2019.

6. Ota A, Tagawa H, Karnan S, Tsuzuki S, Karpas A, Kira S, Yoshida Y and Seto M: Identification and characterization of a novel gene, C13orf25, as a target for 13q31-q32 amplification in malignant lymphoma. Cancer Res 64: 3087-3095, 2004.

7. Doehn C, Böhmer T, Kausch I, Sommerauer M and Jocham D: Prostate cancer vaccines: Current status and future potential. BioDrugs 22: 71-84, 2008.

8. Oesterling JE: Prostate specific antigen: A critical assessment of the most useful tumor marker for adenocarcinoma of the prostate. J Urol 145: 907-923, 1991.

9. Moyer VA and U.S. Preventive Services Task Force: Screening for prostate cancer: U.S. Preventive Services Task Force recommendation statement. Ann Intern Med 157: 120-134, 2012.

10. Tadayon F, Arezegar HR, Khorrami MH, Hashemi Juzdani R, Shahdoost AA and Mellat M: Evaluation of prostatic cancer prevalence in patients with prostatic-specific antigen between 4 and 10 and normal digital rectal examination. Adv Biomed Res 5: 112, 2016.

11. Zugor V, Kühn R, Engelhard K, Poth S, Bernat MM, Porres D and Labanaris AP: The value of endorectal magnetic resonance imaging of the prostate in improving the detection of anterior prostate cancer. Anticancer Res 36: 4279-4283, 2016.

12. Choi SK, Shim M, Kim M, Park M, Lee S, Song C, Lee HL and Ahn H: Heterogeneous oncologic outcomes according to surgical pathology in high-risk prostate cancer: Implications for better risk stratification and preoperative prediction of oncologic outcomes. J Cancer Res Clin Oncol 143: 1871-1878, 2017.

13. Murphy DG and ZargarH:Words of Wisdom.Re: Chemohormonal therapy in metastatic hormone-sensitive prostate cancer. Eur Urol 69: 540, 2016.

14. Langius-Eklöf A, Christiansen M, Lindström V, Blomberg K, Hälleberg Nyman M, Wengström Y and Sundberg K: Adherence to report and patient perception of an interactive app for managing symptoms during radiotherapy for prostate cancer: Descriptive study of logged and interview data. JMIR Cancer 3: e18, 2017.

15. Cheng AM, Byrom MW, Shelton J and Ford LP: Antisense inhibition of human miRNA and indications for an involvement of miRNA in cell growth and apoptosis. Nucleic Acids Res 33: 1290-1297, 2005.

16. $\mathrm{Wu} \mathrm{W,} \mathrm{Sun} \mathrm{M,} \mathrm{Zou} \mathrm{GM} \mathrm{and} \mathrm{Chen} \mathrm{J:} \mathrm{MicroRNA} \mathrm{and} \mathrm{cancer:}$ Current status and prospective. Int J Cancer 120: 953-960, 2007.

17. Iorio MV and Croce CM: MicroRNA dysregulation in cancer: Diagnostics, monitoring and therapeutics. A comprehensive review. EMBO Mol Med 4: 143-159, 2012.

18. Eulalio A, Huntzinger E and Izaurralde E: Getting to the root of miRNA-mediated gene silencing. Cell 132: 9-14, 2008. 
19. Bhayani MK, Calin GA and Lai SY: Functional relevance of miRNA sequences in human disease. Mutat Res 731: 14-19, 2012.

20. Guan B, Wu K, Zeng J, Xu S, Mu L, Gao Y, Wang K, Ma Z, Tian J, Shi Q, et al: Tumor-suppressive microRNA-218 inhibits tumor angiogenesis via targeting the mTOR component RICTOR in prostate cancer. Oncotarget 8: 8162-8172, 2017.

21. Mao A, Liu Y, Wang Y, Zhao Q, Zhou X, Sun C, Di C, Si J, Gan L and Zhang $\mathrm{H}$ : miR-449a enhances radiosensitivity through modulating $\mathrm{pRb} / \mathrm{E} 2 \mathrm{~F} 1$ in prostate cancer cells. Tumour Biol 37 4831-4840, 2016.

22. Liu C, Lv D, Li M, Zhang X, Sun G, Bai Y and Chang D: Hypermethylation of miRNA-589 promoter leads to upregulation of HDAC5 which promotes malignancy in non-small cell lung cancer. Int J Oncol 50: 2079-2090, 2017.

23. Zhu Q, Luo Z, Lu G, Gui F, Wu J, Li F and Ni Y: LncRNA FABP5P3/miR-589-5p/ZMYND19 axis contributes to hepatocellular carcinoma cell proliferation, migration and invasion Biochem Biophys Res Commun 498: 551-558, 2018.

24. Livak KJ and Schmittgen TD: Analysis of relative gene expression data using real-time quantitative PCR and the 2(-Delta Delta C(T)) method. Methods 25: 402-408, 2001.

25. Lin YC, Lin JF, Tsai TF, Chou KY, Chen HE and Hwang TI: Tumor suppressor miRNA-204-5p promotes apoptosis by targeting BCL2 in prostate cancer cells. Asian J Surg 40: 396-406, 2017

26. Wang X, Yi Y, Lv Q, Zhang J, Wu K, Wu W and Zhang W: Novel 1,3,5-triazine derivatives exert potent anti-cervical cancer effects by modulating $\mathrm{Bax}, \mathrm{Bcl} 2$ and caspases expression. Chem Biol Drug Des 91: 728-734, 2018.

27. Powell WC, Knox JD, Navre M, Grogan TM, Kittelson J, Nagle RB and Bowden GT: Expression of the metalloproteinase matrilysin in DU-145 cells increases their invasive potential in severe combined immunodeficient mice. Cancer Res 53: 417-422, 1993.

28. Heidenreich A, Bastian PJ, Bellmunt J, Bolla M, Joniau S, van der Kwast T, Mason M, Matveev V, Wiegel T, Zattoni F, et al: EAU guidelines on prostate cancer. Part 1: Screening, diagnosis, and local treatment with curative intent-update 2013. Eur Urol 65: 124-137, 2014.

29. Veltri RW and Christudass CS: Nuclear morphometry, epigenetic changes, and clinical relevance in prostate cancer. Adv Exp Med Biol 773: 77-99, 2014.

30. He H, Jazdzewski K, Li W, Liyanarachchi S, Nagy R, Volinia S, Calin GA, Liu CG, Franssila K, Suster S, et al: The role of microRNA genes in papillary thyoid carcinoma. Proc Natl Acad Sci USA 102: 19075-19080, 2005.

31. Wu M, Jolicoeur N, Li Z, Zhang L, Fortin Y, L'Abbe D, Yu Z and Shen SH: Genetic variations of microRNAs in human cancer and their effects on the expression of miRNAs. Carcinogenesis 29 : 1710-1716, 2008

32. Zhang X, Jiang P, Shuai L, Chen K, Li Z, Zhang Y, Jiang Y and Li X: miR-589-5p inhibits MAP3K8 and suppresses CD90 ${ }^{+}$ cancer stem cells in hepatocellular carcinoma. J Exp Clin Cancer Res 35: 176, 2016.
33. Monteiro HP, Silva EF and Stern A: Nitric oxide: A potential inducer of adhesion-related apoptosis-anoikis. Nitric Oxide 10: $1-10,2004$.

34. Green DR and Reed JC: Mitochondria and apoptosis. Science 281: 1309-1312, 1998

35. Deveraux QL, Schendel SL and Reed JC: Antiapoptotic proteins The bcl-2 and inhibitor of apoptosis protein families. Cardiol Clin 19: 57-74, 2001.

36. Wang H, Liu G, Shen D, Ye H, Huang J, Jiao L and Sun Y: HOXA1 enhances the cell proliferation, invasion and metastasis of prostate cancer cells. Oncol Rep 34: 1203-1210, 2015.

37. Wang WJ, Yang W, Ouyang ZH, Xue JB, Li XL, Zhang J, He WS Chen WK, Yan YG and Wang C: miR-21 promotes ECM degradation through inhibiting autophagy via the PTEN/akt/mTOR signaling pathway in human degenerated NP cells. Biomed Pharmacother 99: 725-734, 2018.

38. Cui Z, Tang J, Chen J and Wang Z: Hsa-miR-574-5p negatively regulates MACC-1 expression to suppress colorectal cancer liver metastasis. Cancer Cell Int 14: 47, 2014.

39. Bar-Or A, Nuttall RK, Duddy M, Alter A, Kim HJ, Ifergan I, Pennington CJ, Bourgoin P, Edwards DR and Yong VW: Analyses of all matrix metalloproteinase members in leukocytes emphasize monocytes as major inflammatory mediators in multiple sclerosis. Brain 126: 2738-2749, 2003.

40. Westermarck J and Kähäri VM: Regulation of matrix metalloproteinase expression in tumor invasion. FASEB J 13: 781-792, 1999.

41. Kraiem Z and Korem S: Matrix metalloproteinases and the thyroid. Thyroid 10: 1061-1069, 2000.

42. Ventura A and Jacks T: MicroRNAs and cancer: Short RNAs go a long way. Cell 136: 586-591, 2009.

43. Azenshtein E, Luboshits G, Shina S, Neumark E, Shahbazian D, Weil M, Wigler N, Keydar I and Ben-Baruch A: The CC chemokine RANTES in breast carcinoma progression: Regulation of expression and potential mechanisms of promalignant activity. Cancer Res 62: 1093-1102, 2002.

44. Yaal-Hahoshen N, Shina S, Leider-Trejo L, Barnea I, Shabtai EL, Azenshtein E, Greenberg I, Keydar I and Ben-Baruch A: The chemokine CCL5 as a potential prognostic factor predicting disease progression in stage II breast cancer patients. Clin Cancer Res 12: 4474-4478, 2006.

45. Kwon KH, Lee YC, Chung JH and Eun YG: Association study of chemokine (C-C motif) ligand 5 gene polymorphism and papillary thyroid cancer. J Invest Surg 26: 319-324, 2013.

46. Kuo CH, Liu CJ, Lu CY, Hu HM, Kuo FC, Liou YS, Yang YC, Hsieh MC, Lee OK, Wu DC, et al: 17ß-estradiol inhibits mesenchymal stem cells-induced human AGS gastric cancer cell mobility via suppression of CCL5-Src/Cas/Paxillin signaling pathway. Int J Med Sci 11: 7-16, 2013.

47. Swamydas M, Ricci K, Rego SL and Dréau D: Mesenchymal stem cell-derived CCL-9 and CCL-5 promote mammary tumor cell invasion and the activation of matrix metalloproteinases. Cell Adh Migr 7: 315-324, 2013. 